\title{
Effects of Oral Health Care Education on the Severity of Oral Mucositis in Pediatric Oncology Patients
}

\author{
(D) Melahat Akgün KOSTAK', (1) Remziye SEMERCi', (D) Tuba EREN², (D) Esra Nur KOCAASLAN', \\ (1) Filiz YILDIZ ${ }^{2}$
}

'Department of Child Health and Disease Nursing, Trakya University, Faculty of Health Sciences Edirne-Turkey

${ }^{2}$ Department of Paediatric Oncology/Haematology, Trakya University Hospital, Edirne, Turkey

\begin{abstract}
OBJECTIVE
Oral mucositis is undoubtedly the most common painful complication of cancer treatment, especially chemotherapy and adversely affects the quality of life children. This study aimed to examine the effects of oral health care education on the severity of oral mucositis in pediatric oncology patients.

\section{METHODS}

The semi-experimental and longitudinal study was carried out with 30 children aged eight to 18 years and their mothers in the pediatric oncology clinics at a university hospital. Data were collected with Survey Form, World Health Organization Oral Mucositis Index, and Children's International Mucositis Evaluation Scale. Children and mothers in the intervention group received oral health care education before chemotherapy. Children's oral health was assessed before chemotherapy (0th day) and following chemotherapy 7th, 14th, and 21st days. Data were analyzed with descriptive statistics, Mann-Whitney $\mathrm{U}$ test, Friedman Test.
\end{abstract}

\section{RESULTS}

The socio-demographic characteristics of the children did not affect children's oral mucositis severity. It was determined a significant difference in the severity of oral mucositis between the control and intervention group $(\mathrm{p}<0.05)$. Oral mucositis's degree and scores were lower in the intervention groups than the control group on the 7 th day of chemotherapy.

\section{CONCLUSION}

The findings obtained in this study suggest that oral health care education is an effective method to reduce the severity of oral mucositis in pediatric oncology patients. It is recommended that oral health care education should be given to children and their parents from the beginning of hospitalization.

Keywords: Chemotherapy; education; nursing; oral mucositis; pediatric oncology.

Copyright $\odot$ 2020, Turkish Society for Radiation Oncology

\section{Introduction}

Childhood cancer known as cancer in children aged $0-14$ years and for adolescents aged $15-19$ years is a main cause of death in children around the world. [1,2] More than $80 \%$ of childhood cancer cases are reported in lowmiddle nations where access to care and treatment is still insufficient.[1] On the other hand, childhood cancer survival rates have improved along with advanced treatment and medical care.[3] The five-year survival rate for children with cancer is approximately $80-85 \%$ in highincome countries; for Turkey, the rate is $70.8 \%$.[4,5]

Despite advances in cancer treatment, chemotherapy is the most used first option for the type of cancer treatment.[6] Chemotherapy includes the use of chemicals or drugs that kill or prevent the prolifera-

M.Sc. Remziye SEMERCI

Trakya Üniversitesi,

Sağlık Bilimleri Fakültesi,

Çocuk Sağlığı ve Hastalıkları Hemşireliği Anabilim Dalı, Edirne-Turkey

E-mail: remziyesemerci@gmail.com 
tion of cancer cells.[7] While chemotherapy prevents the proliferation and development of cancerous cells, it also damages the mucosal barrier by suppressing the growth and maturation of epithelial cells, which can divide rapidly.[8-10] Oral mucositis is the most common oral problem identified to pediatric oncology patients after the beginning of chemotherapy and is one of the significant side effects of chemotherapy.[11-14] Oral mucositis is occurring in about $52 \%$ to $80 \%$ of children who received cancer treatment.[13]

Oral mucositis is a process leading to disruption in the oral mucosa, erythema, and ulcerative lesions, which are most common in pediatric oncology patients receiving chemotherapy.[14,15] Oral mucositis occurs about a week after intravenous administration of chemotherapies and usually recovery after 21 days. [16] Oral mucositis affects the child's daily life functions, nutrition and quality of life.[15,17] It may also compromise the patient's ability to tolerate planned therapy, can lead to missing doses or reducing doses. Accordingly, it causes prolonged hospital stay and increases the cost of treatment.[9,10,15,17] Moreover, interruptions of treatment and dosage decreases may impair the effectiveness of treatment, which means that the survival, cure and period of remission of patients will be impaired.[11] Therefore, early diagnosis of oral mucositis is important for planning oral care and initiating appropriate treatment.[15]

Nurses should follow evidence-based practices on the subject, to make appropriate interventions according to their planned nursing diagnosis, to monitor and evaluate patients at regular intervals to prevent oral mucositis or to care for its occurrence.[6] Education of the patient and family about oral care is an important role of the nurses. It is important to ensure optimal oral hygiene during treatment as well as adequate nutrition of the patient. MASCC/ISOO guidelines report that the main component of strategies used to decrease the severity of oral mucositis is oral health care protocols and patient education. $[10,18]$ In line with this information, this study aims to investigate the effects of oral health care education on the severity of oral mucositis in pediatric oncology patients.

\section{Hypothesis}

H0; Oral health education which is given to pediatric oncology patients and mothers does not reduce the severity of oral mucositis.

$\mathrm{H} 1$; Oral health education which is given to pediatric oncology patients and mothers reduce the severity of oral mucositis.

\section{Materials and Methods}

\section{Study Design and Sample}

In non-randomized groups, posttest with control group design was used in this study. This design could be used in studies when reaching to the sample size is limited.[19] According to this design, the procedure/ test should be applied only to the intervention group. Then, the effects of the procedure are evaluated by a comparison between the intervention group and the control group.[19]

This semi-experimental and longitudinal study was carried out at the pediatric oncology unit of a university hospital in Edirne, on the northwest of Turkey, between December 2016 and March 2018. It is the only hospital in the Thrace region with a pediatric oncology clinic.

The study population consisted of 8-18 years old children who received chemotherapy treatment. Based on the means and standard deviations oral mucositis scores in the study of Yavuz and Bal Yilmaz (2015), the sample calculation was made in the $G^{\star}$ Power analysis (3.1.9.4) program.[20] The power analysis performed with a power of $99 \%$, effect size $d=2.0$ and alpha at 0.05 , so each group should comprise at least 13 children. Calculating a possible lose sample, the size sample was increased by $20 \%$. Therefore, 15 children were included in each group; thus, the total number of children enrolled was 30 .

The including criteria of this study were 8 - 18 years old patients who received chemotherapy treatment; who had a literacy; who had no oral health problem, no neurodevelopmental delays, verbal difficulties, hearing or visual impairments; had no history of dental oral problem.

\section{Ethical Considerations}

Ethics committee permission was taken Trakya University Ethical Committee of the Faculty of Medicine (Permission no: 21/16) and institution permission was given from the Department of Pediatric Oncology (Permission no: E-105264). This study's aim and the method was explained to mothers and children before this study. The written consent was obtained by the parents and also, verbal assents were obtained by the children. If they did not want to continue working, they were informed that they could withdraw from this study without any reason.

\section{Data Collection Tools}

The sociodemographic data were collected from the children and their mothers with a survey form. The 
oral mucositis degree was evaluated by the children self-report using World Health Organization Oral Mucositis Index and Children's International Mucositis Evaluation Scale (ChIMES).

Survey Form: This form was developed by the researchers as a result of the literature review: 11 questions including socio-demographic characteristics of mothers and children (e.g., age, education level, family structure, family income level and number of children), 11 questions, including oral care and oral mucositis care applications, and the form consists of 22 questions.

World Health Organization (WHO) Oral Mucositis Index: This index is created specifically to measure changes in the grades of oral mucositis related to chemotherapy.[21] The severity of mucositis is assessed on a scale of ' $0=$ No findings-No complaints' to ' $4=$ Bleeding, Necrosis-it does not take solid or liquid food, there is a need for parenteral/enteral nutrition.' depending on the intensity of erythema, oedema, ulceration, and pain.

Children's International Mucositis Evaluation Scale (ChIMES): The scale was developed by Tomlinson et al. (2010) for determining the presence/absence of mucositis in the mouth of children who received chemotherapy [22]. Yavuz et al. (2011) performed Turkish validity reliability of the scale. The scale consists of six items as follows: (1) severity of mouth pain, (2) the effects of pain on swallowing, (3) the effects of pain on eating, (4) the effect of pain on drinking something, (5) pain-relieving condition and reason, (6) presence/absence of intraoral ulcer. Depending on the answer given to each of the 1 st, 2 nd, 3rd and 4 th items of ChIMES, it is evaluated with the lowest ' 0 ' and the highest ' 5 ' points; the fifth item is evaluated with the lowest ' 0 ' and the highest ' 2 ' points; The sixth item is evaluated with the lowest ' 0 ' and the highest ' 1 ' point. The minimum score of scale is 0 , and the maximum score is 23 . The total scores should be measured in the centesimal system for the facility. High scores indicate higher severity of oral mucositis.[23]

\section{Data Collection Procedure}

Before this study, researchers prepared 'Oral health care for the prevention of chemotherapy-induced oral mucositis' presentation with a PowerPoint program for pediatric oncology patients and their mothers separately. Seven experts opinions who were nurses (5) and doctors (2) specialized in pediatric oncology were given for confirming the content of the presentations. The content of the presentation was prepared according to the recommendations of the guidelines.[10,18,24,25] The education topics including; the reason of oral mucositis; pathophysiology of oral mucositis; factors leading to oral mucositis; symptoms of oral mucositis; factors triggering oral mucositis; prevention of oral mucositis; oral and dental care [steps of oral care, medicines to be used for tooth brushing, mouthwash, choosing of the toothbrush, using of electric toothbrushes, using of moisturizer] and nutrition.

Children were included in the groups according to their hospitalization line. Children whose hospitalization line was odd numbers were included in the control group and whose were double numbers were included in the intervention group.

This study was conducted in three steps.

First Step for all Children: The researchers regularly interviewed to chemotherapy nurse to determine chemotherapy day of children. Children were evaluated according to the inclusion criteria by researchers (RS, ENK) before the initiation of chemotherapy (0th day). Eligible children were included in this study. Data were collected face to face interview by 'Survey Form' from all children and their mothers in children's private room at the pediatric oncology unit. All children's mouth was evaluated by 'WHO Oral Mucositis Index' and 'ChIMES. It took 10-15 minutes to complete the data collection tools.

All children and their mothers were administered standard oral care by chemotherapy nurse (FY) during hospitalization.

Standard Oral Care: The standard oral care of pediatric oncology unit was administered to all children by chemotherapy nurse (FY). The standard care was administered to both children and their mothers for daily mouth care. This care included tooth brushing, mouthwash with sodium bicarbonate solution, preparation of sodium bicarbonate solution, oral care with $0.2 \%$ chlorhexidine.

Second Steps for Intervention Group: In additionally standard care, children in the intervention group and their mothers received 'Oral health care education for preventing chemotherapy-induced oral mucositis' by the researcher (RS). The same researcher provided education to all children and their mothers in the intervention group. While the education was given to the mothers in the playroom in the pediatric oncology clinic, the children were given their rooms. The training was given to the mothers separately because the pictures in the presentations could be frightening and stressful for the children. Training took about 2030 minutes. After the presentation, children and their 
mothers were allowed to ask questions about the topics which they did not understand.

Third Steps for all Children: All children's mouth evaluated 0 th, 7 th, 14 th and 21 st days of chemotherapy with 'WHO Oral Mucositis Index' and 'ChIMES' by the researchers (RS, ENK).

\section{Data Analysis}

Data were analyzed using SPSS 23 package program (Statistical Package for the Social Science). Descriptive statistics which are mean, standard deviation, frequency and percentage were used analysis of independent variables. The distribution of continuous variables was analyzed using the Shapiro Wilks test. The Friedman test was used for the comparison of independent and dependent variables. Mann-Whitney $U$ test was used for evaluating the source of differences. The results were evaluated in a confidence interval of $95 \%$, and $\mathrm{p}<0.05$ was considered significant.

\section{Results}

\section{Characteristics of Children}

According to the groups, the characteristics of the children and mothers showed a homogeneous distribution. The mean age of the children was $12.87 \pm 3.02$ years in the control group, $12.46 \pm 3.27$ years in the intervention group. More of the children (66.7\%) were male in the control group, $53.3 \%$ of the intervention group were female. The mean of diagnosis time was $12.87 \pm 19.76$ months for the control group, $14.27 \pm$ 21.89 months for the intervention group. Most of the children were diagnosed with ALL (53.3\% of intervention; $40 \%$ of control). More of the children were at the second phase of diseases (46.7\% of control; $40.0 \%$ of intervention). Most of the children received third or fourth chemotherapy cure (75.0\% of the control group; $66.7 \%$ of the intervention group). There was no significant difference between the socio-demographic characteristics of the groups and mean of the oral mucositis scores $(\mathrm{p}>0.05)$.

The mean age of the mothers was $39.53 \pm 5.68$ years in the control group, $40.80 \pm 5.89$ years in the intervention group. More of the mothers graduated from high school (53.3\% of control; $40.0 \%$ of intervention). All of the mothers were unemployed. Both groups, most of them were nuclear families and had moderate income.

It was found that $66.7 \%$ of the control group and $53.3 \%$ of the intervention group brush their teeth twice times in a day, most of the children practice oral care four times in a day (93.3\% of control; $86.7 \%$ of inter- vention). All of the children received information about mouth care from nurses; however, only half of them received information about oral mucositis from nurses ( $66.7 \%$ of control; $53.3 \%$ of intervention).

\section{Mucositis Severity of children}

Children's level of oral mucositis was evaluated with the WHO Oral Mucositis Index, there was a significant difference among groups $(\mathrm{p}<0.001)$. The difference was found that children in control group (Table 1; Fig. 1). In the advanced analysis, the oral mucositis levels of the children were compared according to days, there was a significant difference between the groups on the seventh day $(p=0.014)$. The children in the control group had a higher level of oral mucositis than the intervention group on the seventh day.

Children's level of oral mucositis was evaluated with ChIMES, there was a significant difference among groups ( $\mathrm{p}=0.005)$. The significant difference was found in the control group (Table 2; Fig. 2). In the advanced analysis, the oral mucositis levels of the children were compared according to days, there was a significant difference between the groups on the seventh day $(\mathrm{p}=0.027)$. The children in the control group had a higher level of oral mucositis than the intervention group on the seventh day.

\section{Discussion}

Oral mucositis is undoubtedly the most common symptom of cytotoxic treatment, especially chemotherapy. [26,27] Oral mucositis could be caused hospitalization by causing swallowing, eating and drinking disorders, and as a result, the quality of life of patients might be decreased.[28] Despite medical treatment, hospitalization may be prolonged, which may lead to increased risk of infection and increased hospital costs.[23,24,27] The incidence of oral mucositis in children is higher compared to adults due to the high rate of regeneration of mucous cells in children, and the change in immunological response and resistance.[23] Thus, early diagnosis of mucositis is crucial for developing an oral care plan and initiating appropriate treatment. While many guidelines clearly state that there is lack of evidence that oral care procedures and education for preventing oral mucositis, it is also emphasized that patient education is important for reducing the incidence of oral mucositis caused by cytotoxic drugs.[10,18,24,25] Thus, the present study aimed to investigate the effects of oral health care education which is given pediatric oncology patients and their mother on the severity of 


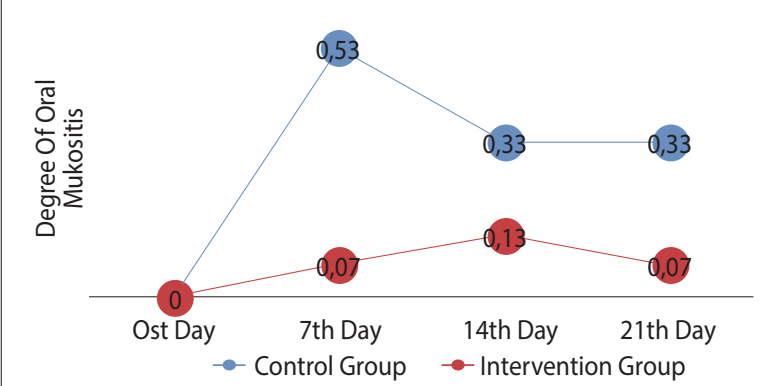

Fig. 1. Children's oral mucositis degree according to the WHO oral mucositis index.

oral mucositis. It was determined that oral health care education is effective for reducing severity of oral mucositis in pediatric oncology patients.

This is widely accepted that oral care is a significant complement to the prevention, severity and management of oral mucositis.[27] Patients are not regularly informed about how to perform oral care and the symptoms of oral mucositis, so healthcare professionals should be educated about the benefits of oral health to their parents and children, but this is often overlooked in the clinic. In this study, it was found that al-

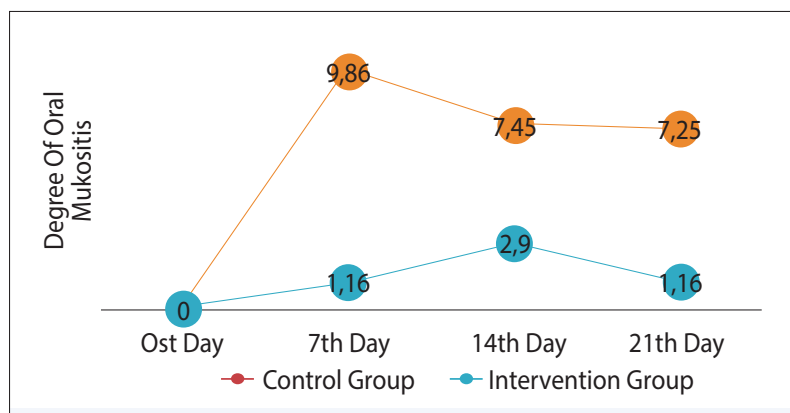

Fig. 2. Children's oral mucositis degree according to ChIMES.

though all the children received information about oral care, nearly half of them did not receive information about oral mucositis. In a study conducted by Yavuz and Bal Yllmaz, it was reported that $68.75 \%$ of children with cancer received mouth care education and $75.0 \%$ of them received education related to oral mucositis. [15] Arshadi Bostanabad et al. stated that $56.6 \%$ of the pediatric oncology patients did not receive education about oral mucositis.[29] Nurses play a critical role in the prevention and management of oral mucositis. Also, patient education is an important component of

Table 1. The distribution of mucositis severity according to WHO oral mucositis index

\begin{tabular}{|c|c|c|c|c|c|c|c|c|}
\hline & \multicolumn{3}{|c|}{ Control Group ( $n=15)$} & \multicolumn{3}{|c|}{ Intervention Group $(n=15)$} & \multirow[t]{2}{*}{ Test* } & \multirow[t]{2}{*}{$\mathbf{p}$} \\
\hline & $\mathrm{M} \pm \mathrm{SD}$ & Min & Max & $\mathrm{M} \pm \mathrm{SD}$ & Min & Max & & \\
\hline Day 0th & $0.00 \pm 0.00$ & 0.00 & 0.00 & $0.00 \pm 0.00$ & 0.00 & 0.00 & 112.500 & 1.000 \\
\hline Day 7th & $1.13 \pm 1.46$ & 0.00 & 4.00 & $0.13 \pm 0.52$ & 0.00 & 2.00 & 66.500 & 0.014 \\
\hline Day 14th & $0.33 \pm 0.62$ & 0.00 & 2.00 & $0.13 \pm 0.35$ & 0.00 & 1.00 & 96.500 & 0.340 \\
\hline Day 21st & $0.33 \pm 0.61$ & 0.00 & 2.00 & $0.07 \pm 0.26$ & 0.00 & 1.00 & 89.500 & 0.141 \\
\hline Test** & & 19.000 & & & 3.000 & & & \\
\hline $\mathrm{p}$ & & $<0.001$ & & & 0.392 & & & \\
\hline
\end{tabular}

* Mann-Whitney U; ** Friedman Test; M: Mean; SD: Standard Deviation

Table 2. The distribution of mucositis severity according to ChIMES

\begin{tabular}{|c|c|c|c|c|c|c|c|c|}
\hline & \multicolumn{3}{|c|}{ Control Group ( $n=15)$} & \multicolumn{3}{|c|}{ Intervention Group $(n=15)$} & \multirow[t]{2}{*}{ Test* } & \multirow[t]{2}{*}{$\mathbf{p}$} \\
\hline & $M \pm S D$ & Min & Max & $\mathrm{M} \pm \mathrm{SD}$ & Min & Max & & \\
\hline Day 0th & $0.00 \pm 0.00$ & 0.00 & 0.00 & $0.00 \pm 0.00$ & 0.00 & 0.00 & 112.500 & 1.000 \\
\hline Day 7th & $11.01 \pm 47.83$ & 0.00 & 47.83 & $1.16 \pm 4.49$ & 0.00 & 17.39 & 73.000 & 0.027 \\
\hline Day 14th & $7.45 \pm 12.82$ & 0.00 & 30.43 & $2.90 \pm 7.82$ & 0.00 & 26.09 & 94.500 & 0.285 \\
\hline Day 21st & $7.25 \pm 12.48$ & 0.00 & 30.43 & $1.16 \pm 4.49$ & 0.00 & 17.39 & 88.000 & 0.117 \\
\hline Test** & & 12.900 & & & 4.000 & & & \\
\hline $\mathrm{p}$ & & 0.005 & & & 0.261 & & & \\
\hline
\end{tabular}

\footnotetext{
* Mann-Whitney U; ** Friedman Test; M: Mean; SD: Standard Deviation
} 
care based on collaboration with the healthcare team within the patient's needs.[30] In a study carried out by Özcan Yüce and Yurtsever, it was found that education about oral mucositis reduced patients degree of oral mucositis and increased the quality of life.[31] Therefore, nurses and health care professionals should educated pediatric oncology patients and parents about oral care/oral mucositis for preventing and reducing the severity of oral mucositis.

In this study, the findings showed that children in the intervention group had a lower severity of oral mucositis than the control group on 7 th chemotherapy day. Oral mucous membranes are usually damaged between seven and fourteen days following cytotoxic therapy by the subjective complaint of pain, burning sensation or sorrow.[32] Cancer patients receiving chemotherapy generally become symptomatic four or five days after beginning chemotherapy, reaching a peak of on 10th day, and then slowly recovering in several weeks.[26] If the occurrence of oral mucositis can be prevented or reduced severity in the first week after treatment, the formation will be prevented in the next period. Also, the results of this research are in line with the literature. Yavuz and Bal Yilmaz examined the effects of oral care education on the severity of oral mucositis in pediatric oncology patient, and it was reported that children who received education had a lower oral mucositis' degree on the 5th, 7th, 10th day of chemotherapy.[15] Thus, it can be said that oral care education is an effective method to prevent and reduce the severity of oral mucositis among pediatric oncology patients.

While oral mucositis is not a life-threatening complication, it has a significant impact on children's clinic features and may have a wide range of physical and psychosocial consequences for patients.[33] Thus, nurses should be aware of the effects of oral mucositis on children and they should inform children and their parents about oral care techniques for the prevention and management of mucositis from the beginning of hospitalization.

This study had some limitations. Firstly, this study was performed within a single centre. Secondly, all of the pediatric cancer types and all treatment types which may influence different symptom were included in this study, maybe future study may be limited with some protocols or diseases. Despite these limitations, the study' effect size is high $\mathrm{d}=1.98$. Therefore, study results provide important baseline information about the effects of oral health care for reducing the severity of oral mucositis.

\section{Conclusion}

The findings obtained in this study suggest that oral health care education which is given to pediatric oncology patients and their mothers to prevent oral mucositis induced chemotherapy is effective for reducing the severity of mucositis in pediatric oncology patients. Therefore, we recommend that pediatric cancer patients and their mothers should be informed about oral care and oral mucositis to prevent and reduce the severity of oral mucositis from the first day of hospitalization. It was also recommended that nurses should assess the oral health of children every day.

Acknowledgments: We also thankful to the pediatric oncology healthcare professionals for their collaborations.

Peer-review: Externally peer-reviewed.

Conflict of Interest: All authors declared no conflict of interest.

Ethics Committee Approval: Ethics committee permission was taken Trakya University Ethical Committee of the Faculty of Medicine (Permission no: 21/16).

Financial Support: The authors have no funding or conflicts of interest to disclose.

Authorship contributions: Concept - R.S; Design - R.S; M.A.K.; Supervision - R.S; E.N.K; Materials - R.S; E.N.K ; Data collection \&/or processing - R.S; E.N.K; F.Y. ; Analysis and/or interpretation - R.S; M.A.K; Literature search - R.S; E.N.K; Writing - R.S; M.A.K; Critical review -M.A.K; T.E.

\section{References}

1. Ward ZJ, Yeh JM, Bhakta N, Frazier AL, Atun R. Estimating the total incidence of global childhood cancer: a simulation-based analysis. Lancet Oncol 2019;20(4):483-93.

2. Autier P. Increasing incidence of cancer in children and competing risks. Lancet Oncol 2018;19(9):1136-7.

3. Kaya C, Chan F, Tansey T, Bezyak J, Aksoy S, Altundag K. Evaluating the World Health Organization's International Classification of Functioning, Disability and Health Framework as a Participation Model for Cancer Survivors in Turkey. Rehabil Couns Bull 2019;62(4):222-33.

4. Kutluk T, Yeşilipek A. Pediatric cancer registry in Turkey 2009-2018 (TPOG \& TPHD). J Clin Oncol 2019;37(15):21510.

5. B Bhakta N, Force LM, Allemani C, Atun R, Bray F, Coleman MP, et al. Childhood cancer burden: a review of global estimates. Lancet Oncol 2019;20(1):42-53. 
6. Erden Y, Ipekcoban G. Comparison of efficacy of cryotherapy and chlorhexidine to oral nutrition transition time in chemotherapy-induced oral mucositis. Eur J Cancer Care (Engl) 2017;26(5).

7. Carreón-Burciaga RG, Castañeda-Castaneira E, González-González R, Molina-Frechero N, Gaona E, Bologna-Molina R. Severity of Oral Mucositis in Children following Chemotherapy and Radiotherapy and Its Implications at a Single Oncology Centre in Durango State, Mexico. Int J Pediatr 2018;2018:3252765.

8. Çıtlak K, Kapucu S. Current approaches to oral mucositis prevention and treatment in patients receiving chemotherapy: Evidence-based practices. Journal of Hacettepe University Faculty of Nursing 2015;2(1):70-7.

9. Sonis ST. Mucositis: The impact, biology and therapeutic opportunities of oral mucositis. Oral Oncol 2009;45(12):1015-20.

10. Lalla RV, Bowen J, Barasch A, Elting L, Epstein J, Keefe DM, et al. MASCC/ISOO clinical practice guidelines for the management of mucositis secondary to cancer therapy. Cancer 2014;120(10):1453-61.

11. Allen G, Logan R, Revesz T, Keefe D, Gue S. The Prevalence and Investigation of Risk Factors of Oral Mucositis in a Pediatric Oncology Inpatient Population; a Prospective Study. J Pediatr Hematol Oncol 2018;40(1):15-21.

12. Ribeiro ILA, Melo ACR, Limão NP, Bonan PRF, Lima Neto EA, Valença AMG. Oral Mucositis in Pediatric Oncology Patients: A Nested Case-Control to a Prospective Cohort. Braz Dent J 2020;31(1):78-88.

13. He M, Zhang B, Shen N, Wu N, Sun J. A systematic review and meta-analysis of the effect of low-level laser therapy (LLLT) on chemotherapy-induced oral mucositis in pediatric and young patients. Eur J Pediatr 2018;177(1):7-17.

14. Parra JJ, Alvarado MC, Monsalve P, Costa ALF, Montesinos GA, Parra PA. Oral health in children with acute lymphoblastic leukaemia: before and after chemotherapy treatment. Eur Arch Paediatr Dent 2020;21(1):129-36.

15. Yavuz B, Bal Yulmaz H. Investigation of the effects of planned mouth care education on the degree of oral mucositis in pediatric oncology patients. J Pediatr Oncol Nurs 2015;32(1):47-56.

16. Ünal Çubukçu N, Çınar S. Can oral mucositis be prevented in patients receiving chemotherapy?. Journal of Marmara University Institute of Health Sciences 2012;2(4):155-63.

17. Yllmaz MÇ. Evidence-based practices for managing mucositis. Int J Hematol Oncol 2007;4(17):241-6.

18. Bowen JM, Elad S, Hutchins RD, Lalla RV, Mucositis Study Group of the Multinational Association of Sup- portive Care in Cancer/International Society of Oral Oncology (MASCC/ISOO). Methodology for the MASCC/ISOO Mucositis Clinical Practice Guidelines Update. Support Care Cancer 2013;21(1):303-8.

19. Nahcivan N. Nicel. Araştırma tasarımları. In: Erdoğan S, Nahcivan N, Esin N, editors. Hemşirelikte araştırma, süreç, uygulama ve kritik. İstanbul: Nobel Tip Kitabevleri; 2015. p. 117-20.

20. Faul F, Erdfelder E, Lang AG, Buchner A. G*Power 3: a flexible statistical power analysis program for the social, behavioral, and biomedical sciences. Behav Res Methods 2007;39(2):175-91.

21. World Health Organisation. WHO Handbook for reporting results of cancer treatment. Geneva: WHO Offset Publications; 1979.

22. Tomlinson D, Gibson F, Treister N, Baggott C, Judd P, Hendershot E, et al. Refinement of the Children's International Mucositis Evaluation Scale (ChIMES): child and parent perspectives on understandability, content validity and acceptability. Eur J Oncol Nurs 2010;14(1):29-41.

23. Yavuz B, Bal Yilmaz H, Karaman N. A study of reliability and validity for the Turkish version of children's international mucositisis evaluation scale for children with cancer. Turk J Oncol 2011;26(4):157-62.

24. Qutob AF, Gue S, Revesz T, Logan RM, Keefe D. Prevention of oral mucositis in children receiving cancer therapy: a systematic review and evidence-based analysis. Oral Oncol 2013;49(2):102-7.

25. Israels T, Renner L, Hendricks M, Hesseling P, Howard S, Molyneux E, et al. SIOP PODC: recommendations for supportive care of children with cancer in a low-income setting. Pediatr Blood Cancer 2013;60(6):899-904.

26. Mishra L, Nayak G. Effect of flavored (honey and tulsi) ice chips on reduction of oral mucositis among children receiving chemotherapy. Int J Pharm Sci Rev Res 2017;43:25-8.

27. Hogan R. Implementation of an oral care protocol and its effects on oral mucositis. J Pediatr Oncol Nurs 2009;26(3):125-35.

28. Qutob AF, Allen G, Gue S, Revesz T, Logan RM, Keefe D. Implementation of a hospital oral care protocol and recording of oral mucositis in children receiving cancer treatment : a retrospective and a prospective study. Support Care Cancer 2013;21(4):1113-20.

29. Arshadi Bostanabad M, Alvandnezhad T, Hiradfar A, Mohammadpoorasi A, Khalvati B. Level of education provided by nurses to the parents of children with chemotherapy-induced mucositis. Journal of Clinical and Basic Research 2018;2(2):29-34.

30. Perry AD, Iida H, Patton LL, Wilder RS. Knowledge, Perceived Ability and Practice Behaviors Regarding 
Oral Health among Pediatric Hematology and Oncology Nurses. J Dent Hyg 2015;89(4):219-28.

31. Yüce UÖ, Yurtsever S. Effect of Education About Oral Mucositis Given to the Cancer Patients Having Chemotherapy on Life Quality. J Cancer Educ 2019;34(1):35-40.

32. Singh R, Sharma S, Kaur S, Medhi B, Trehan A, Bijarania SK. Effectiveness of Topical Application of Honey on Oral Mucosa of Children for the Management of Oral Mucositis Associated with Chemotherapy. Indian J Pediatr 2019;86(3):224-8.

33. Cheng KK, Chang AM, Yuen MP. Prevention of oral mucositis in paediatric patients treated with chemotherapy; a randomised crossover trial comparing two protocols of oral care. Eur J Cancer 2004;40(8):1208-16. 